\title{
A prática do acolhimento na atenção ao usuário de substâncias psicoativas: percepção do usuário
}

\section{The practice of embrancement on attention to the user of psychoactive substances: user perception}

\author{
Meire Luci da Silva ${ }^{1}$, Luiza Dorini Marucci², Caroline da Silva Guimarães ${ }^{3}$ \\ http://dx.doi.org/10.11606/issn.2238-6149.v30i1p1-9
}

\begin{abstract}
Silva M, Marucci LD, Guimarães CS. A prática do acolhimento na atenção ao usuário de substâncias psicoativas: percepção do usuário. Rev Ter Ocup Univ São Paulo. 2019 jan.-abr.;30(1):1-9.
\end{abstract}

RESUMO: Este artigo tem como objetivo investigar a percepção do usuário de substâncias psicoativas em tratamento, acerca do acolhimento recebido em Centros de Atenção Psicossocial ao Usuário de Álcool e Outras Drogas (CAPSad). Pesquisa quantiqualitativa realizada em CAPSad de quatro cidades do interior paulista. Como instrumento investigativo utilizou-se um questionário semiestruturado com 26 questões. Para análise das respostas abertas foi utilizado método de análise de conteúdo e, estatística descritiva para respostas fechadas. Os resultados permitiram aprofundar conhecimentos sobre a realidade da prática do acolhimento ao usuário de substâncias, apontando para diversidades e dificuldades sobre o dispositivo, o que pode restringir a utilização deste pelo usuário em sua totalidade de direito, mesmo que este reconheça a importância do acolhimento durante o processo de tratamento. Destaca-se necessidade de desenvolvimento de ações e políticas informativas sobre o dispositivo para o usuário. Também se destaca a necessidade de investimento na capacitação e sensibilização dos profissionais para utilização do dispositivo na construção de relações de cuidados mais próximas e efetivas.

DESCRITORES: Acolhimento; Transtornos relacionados ao uso de substâncias; Humanização da assistência.
Silva M, Marucci LD, Guimarães CS. The practice of embrancement on attention to the user of psychoactive substances: user perception. Rev Ter Ocup Univ São Paulo. 2019 Jan.-Apr.;30(1):1-9.

ABSTRACT: This article aimed to investigate the perception of the user of psychoactive substances in treatment, about the reception received in Centers of Psychosocial Attention to the User of Alcohol and Other Drugs (CAPSad). Quantiqualitative research carried in CAPSad of four cities in the State of São Paulo. As investigative tool we used a semi-structured questionnaire with 26 questions. For the analysis of the open answers we used the content analysis method and descriptive statistics for closed answers. The results allowed to deepen knowledge on the reality of the practice of the reception to the user of substances, pointing to diversities and difficulties about the device, which may restrict the use of this by the user in its entirety of right, even if the user acknowledges the importance of the host during the treatment process. It is highlighted the need to develop actions and information policies on the device for the user. It also highlights the need for investment in the training and awareness of professionals to use the device in the construction of closer and more effective care relationships.

KEYWORDS: User embracement; Substance-related disorders; Humanization of assistance.

Recorte de projeto maior intitulado “Acolhimento na atenção à Dependência Química”. Apresentado no $15^{\circ}$ Congresso Brasileiro de Terapia Ocupacional, Porto Alegre, out. 2017. XXIX Congresso de Iniciação Cientifica da UNESP, Marília, set. 2017.

1. Professora Assistente Doutora do curso de Terapia Ocupacional do Departamento de Fisioterapia e Terapia Ocupacional da Faculdade de Filosofia e Ciências da Universidade Estadual Paulista (UNESP), Marilia, SP, Brasil. Tutora do Programa de Residência Integrada Multiprofissional em Saúde Mental da Faculdade de Medicina de Marília (FAMEMA), Marilia, SP, Brasil. ORCID: https://orcid.org/00000003-0256-4793. Email: meire.silva@unesp.br.

2. Terapeuta Ocupacional, Residente em Programa Multiprofissional em Saúde Mental da Faculdade de Medicina de Marília - FAMEMA, Marília, SP, Brasil. ORCID: https://orcid.org/0000-0001-7413-590X. Email: marucciluiza@gmail.com.

3. Discente do curso de Terapia Ocupacional da Faculdade de Filosofia e Ciências da Universidade Estadual Paulista - UNESP, Marília, SP, Brasil. ORCID: https://orcid.org/0000-0002-1616-8962.Email: carol.guimaraes_@hotmail.com.

Endereço para correspondência: Avenida Hygino Muzzi Filho, 737 - Bairro Mirante, Marília, São Paulo, Brasil. CEP: 17525-000. Email: meire.silva@unesp.br. 


\section{INTRODUÇÃO}

A tualmente, o uso, abuso e dependência de problema de saúde pública, que devido ao seu caráter multifatorial exige inúmeros esforços e ações voltadas ao tratamento, bem como prevenção e promoção de saúde e de defesa da vida do usuário. Uma vez já instalado, o transtorno relacionado ao uso de substâncias afeta significativamente todas as dimensões da vida do usuário. Portanto, para traçar as ações de cuidado e atenção ao usuário é necessário compreender o papel e significado da substância na vida deste. E, para o sucesso das ações, estas devem ser pautadas na singularidade, subjetividade, contextos e valores de vida de cada usuário ${ }^{1}$.

Nesta perspectiva e, visando a atenção e cuidado à saúde integral do indivíduo em sofrimento psíquico e ao usuário de substâncias psicoativas, o Sistema Único de Saúde (SUS) implantou a Rede de Atenção Psicossocial (RAPS). Esta rede consiste em estratégias e dispositivos de atenção, distribuídas entre a atenção básica, psicossocial, urgência e emergência, de caráter transitório, hospitalar e estratégias de desinstitucionalização e reabilitação psicossocial ${ }^{2}$.

Dentre os serviços da RAPS na atenção psicossocial ao usuário de substâncias destaca-se os Centros de Atenção Psicossocial a usuários de Álcool e outras Drogas (CAPSad), que foram instituídos como serviços de referência para este público ${ }^{3}$. Dentre as práticas profissionais desenvolvidas nos CAPSad, destacam-se o acolhimento, atendimentos individuais (medicamentoso, de orientação, psicoterápico, entre outros), em grupos, oficinas terapêuticas e visitas domiciliares.

Com a Política de Saúde Mental, Álcool e Outras Drogas (PNAD) instituída pelo Ministério da Saúde ${ }^{3}$, os profissionais dos CAPSad deveriam ter suas ações e intervenções norteadas pelos princípios da Política de Redução de Danos, a qual tinha como diretriz, a diminuição dos prejuízos e dos riscos sociais e à saúde do usuário e da comunidade, decorrentes do uso abusivo de substâncias ${ }^{4}$. Em início de 2018 foram definidas novas diretrizes para realinhamento e fortalecimento das ações da PNAD, devendo os programas, projetos e ações promover a ampliação e reorganização da rede de cuidados, acolhimento e suporte social, baseados na prevenção, promoção da saúde e da abstinência de substâncias, bem como suporte social e redução dos riscos sociais e de saúde dos usuários ${ }^{5}$.

Para efetividade da atenção ao usuário, o planejamento das estratégias de cuidado e projetos terapêuticos deve considerar não só a singularidade do usuário, mas também o contexto biopsicossocial, econômico e cultural no qual está inserido, além de que, devem ser elaboradas juntamente com o usuário, família e comunidade ${ }^{1}$. O planejamento das ações em conjunto com os atores envolvidos no processo do cuidado possibilita ao usuário, o exercício do protagonismo de vida, além da sua corresponsabilização do tratamento.

Todos os serviços de atenção à saúde, inclusive CAPSad também devem ter suas práticas pautadas nas diretrizes da Política Nacional de Humanização (HumanizaSUS), que propõe a prática do cuidado humanizado através de atendimentos que vise o ser humano em sua integralidade, complexidade e no desempenho de diferentes papéis sociais ${ }^{6}$.

Neste contexto, o acolhimento deve ser o eixo norteador das práticas dos profissionais do CAPSad, consistindo no desenvolvimento de prática profissional e de postura ética, através da escuta qualificada de suas queixas, preocupações e angústias. A prática do acolhimento possibilita aos profissionais, analisar a demanda do usuário e possíveis encaminhamentos futuros, bem como elaboração de projeto terapêutico singular ${ }^{6}$.

Dentre os motivos que podem dificultar a busca e adesão ao tratamento pelo usuário estão: dificuldade em perceber as consequências negativas do uso e prejuízos destes sentimentos de ambivalência em relação à substância, falta de aceitação da dependência, falta de motivação para cessar o uso, dificuldade em relação às mudanças comportamentais ${ }^{7}$. Para dificultar ainda mais este processo atrela-se a dificuldade de acesso e receio quanto à forma de recepção nos serviços e o estigma relacionado ao transtorno. Frente a estes motivos, a chegada e permanência do usuário no serviço, podem ser consideradas situações de difícil manejo e, é na perspectiva de "estar perto de" ou "estar com" que a prática do acolhimento possui na ação, seu real e prático significado.

Nesta perspectiva, o acolhimento pode ser dispositivo facilitador não só da entrada do usuário ao serviço, mas também de sua deambulação, permanência e adesão ao tratamento, consistindo em estratégia para criação e fortalecimento de vínculos, estimulando e facilitando a inclusão gradual do usuário no serviço e no tratamento ${ }^{8,9}$.

O acolhimento, portanto, é um dispositivo de extrema importância durante o tratamento do usuário, devendo estar presente em todos os momentos e, funcionando na maioria das vezes, como "porta de entrada" para o tratamento ${ }^{4}$. Por considerar a importância da humanização nas relações de cuidado, a função e importância do acolhimento no processo de tratamento, o objetivo desta pesquisa foi investigar a percepção do usuário de substâncias psicoativas em tratamento, acerca do acolhimento recebido em Centros de Atenção Psicossocial ao Usuário de Álcool e Outras Drogas (CAPSad). 


\section{METODOLOGIA}

Trata-se de uma pesquisa descritiva, qualitativa e de caráter exploratório. A pesquisa foi realizada em quatro Centros de Atenção Psicossocial ao Usuário de Álcool e Outras Drogas II (CAPSad) de quatro cidades do interior paulista. Ressalta-se que devido a aspectos éticos, a fim de garantir o anonimato do serviço, optou-se pela não identificação das cidades onde estavam localizados os CAPSad. No ano de 2015, havia 65 CAPSad em funcionamento no estado de São Paulo ${ }^{10}$, porém a escolha dos CAPSad II para pesquisa foi devida proximidade em relação às suas localizações geográficas e, portanto, conveniência.

Foram convidados 83 usuários, destes nove recusaram. Portanto, a amostra foi composta por 74 usuários de substâncias psicoativas, diagnosticados com transtorno relacionado ao uso de substâncias e em tratamento. Como critérios de inclusão, o usuário deveria aceitar participar voluntariamente da pesquisa, ser maior de 18 anos, apresentar funções cognitivas preservadas segundo avaliação médica, realizar tratamento no CAPSad e estar abstinente no momento da coleta. Os critérios de exclusão foram: ser menor de 18 anos, não possuir diagnóstico, apresentar comprometimento de funções cognitivas, não realizar tratamento no CAPSad e estar sob efeito de alguma substância no momento da coleta. As justificativas dos nove participantes que se recusaram a participar foram: falta de disponibilidade de tempo e falta de conhecimento sobre a temática da pesquisa.

Esta pesquisa cumpriu todos os aspectos éticos, tendo sido aprovada pelo Comitê de Ética em Pesquisa sob parecer 1.399.315 no ano de 2016. Todos os participantes foram informados sobre o objetivo da pesquisa e da importância de sua participação, bem como sigilo e anonimato. Após concordância e consentimento em participar, todos assinaram o Termo de Consentimento Livre e Esclarecido (TCLE).

Como instrumento de pesquisa, foi elaborado e aplicado questionário de autopreenchimento com 26 questões, sendo 17 fechadas e nove abertas. $\mathrm{O}$ instrumento foi composto por duas partes, sendo: caracterização do perfil socioeconômico e caracterização do tratamento atual e do acolhimento recebido. Em etapa anterior à aplicação, foi realizado teste piloto com amostra reduzida de três usuários. Estes avaliaram o instrumento quanto à fidedignidade, clareza dos enunciados, compreensão do significado e acessibilidade ao vocabulário do conteúdo das questões. A partir dos apontamentos dos avaliadores foram realizados pequenos ajustes.

Para coleta dos dados, foi realizado contato prévio com a gerência de cada CAPSad e, posteriormente com os profissionais do serviço para explanação da proposta de pesquisa. Após aprovação destes, foi estabelecido pelas equipes dos serviços que a explicação sobre o conteúdo e convite para participação na pesquisa deveria ser realizado coletivamente, em momentos iniciais de atividades grupais oferecidas pelos serviços. Após demonstração de interesse do usuário em participar do estudo, foi agendado data e hora para aplicação do instrumento e assinatura do TCLE.

A coleta de dados foi realizada de agosto de 2016 a junho de 2017. Os testes foram aplicados individualmente, tiveram duração média de 20 minutos e foram acompanhados pelo estudante de iniciação científica devidamente treinado pelo pesquisador responsável. No caso, de auxílio na leitura, realizava a releitura junto ao usuário, evitando interpretações das questões e, possíveis interferências nas respostas.

Todos os resultados foram digitados em planilha do Microsoft Excel 2010 e, posteriormente transferidos para o programa estatístico Statistical Package for the Social Sciences 17.0 (SPSS). Para análise das variáveis quantitativas aplicou-se cálculos de estatística descritiva, como: frequência, média, desvio padrão e porcentagem. Para análise das variáveis qualitativas foi utilizado o método de análise de conteúdo ${ }^{11}$. As respostas foram transcritas integralmente, analisadas e categorizadas por temáticas.

\section{RESULTADOS}

\section{Caracterização socioeconômica da amostra}

Participaram 74 usuários, com idade média de 42,4 anos (DP $\pm 11,13)$, sendo que $83,7 \%$ eram participantes do sexo masculino. Na Tabela 1, é possível verificar a caracterização socioeconômica dos participantes.

Tabela 1 - Características do perfil socioeconômico dos participantes

\begin{tabular}{llcc}
\hline \multirow{2}{*}{ Variáveis } & Categoria & $\begin{array}{c}\text { Frequência } \\
\text { (n) }\end{array}$ & $\begin{array}{c}\text { Porcentagem } \\
\text { (\%) }\end{array}$ \\
\hline \multirow{2}{*}{ Sexo } & Feminino & 12 & $16,2 \%$ \\
& Masculino & 62 & $83,8 \%$ \\
& Sem companheiro (a) & & \\
& (solteiro/divorciado/ & 51 & $68,9 \%$ \\
Estado civil & viúvo) & & \\
& Com companheiro (a) & & \\
& (Casado/ união & 23 & $31,1 \%$ \\
\multirow{5}{*}{ Religião } & estável) & & \\
& Possui & 66 & $89,1 \%$ \\
& Não possui & 08 & $10,9 \%$ \\
\multirow{5}{*}{ Escolaridade } & Não alfabetizado & 03 & $04,0 \%$ \\
& Ensino Fundamental & 33 & $44,6 \%$ \\
& Ensino médio & 27 & $36,5 \%$ \\
& Ensino superior & 11 & $14,9 \%$ \\
\hline & & & Continua $\ldots$
\end{tabular}


Silva M, et al. A prática do acolhimento na atenção ao usuário de substâncias psicoativas. Rev Ter Ocup Univ São Paulo. 2019 jan./abr.;30(1):1-9.

Tabela 1 - Características do perfil socioeconômico dos participantes

\begin{tabular}{llcc}
\hline Variáveis & Categoria & $\begin{array}{c}\text { Frequência } \\
\text { (n) }\end{array}$ & $\begin{array}{c}\text { Porcentagem } \\
\text { (\%) }\end{array}$ \\
\hline \multirow{2}{*}{ Renda } & Não possui & & \\
financeira & Menos de 2 salários & 13 & $17,5 \%$ \\
familiar & De 3 ou mais salários & 43 & $58,1 \%$ \\
& mínimos & 01 & $23,0 \%$ \\
& Não respondeu & & $01,4 \%$ \\
Auxílio & Recebe & 24 & $32,4 \%$ \\
Financeiro & Não recebe & 48 & $64,9 \%$ \\
do Estado & Não respondeu & 02 & $02,7 \%$ \\
Despesas & Contribui & 54 & $73,0 \%$ \\
Mensais & Não contribui & 18 & $24,3 \%$ \\
& Não respondeu & 02 & $02,7 \%$ \\
\hline
\end{tabular}

Fonte: Própria

\section{Caracterização do tratamento e acolhimento recebido}

Quanto à decisão de iniciar tratamento, 75,7\% (56) responderam decisão própria, $13,5 \%$ (10) de familiares e $10,8 \%$ (08) decisão por outros (profissionais da área médica e justiça). Já em relação ao tempo de tratamento atual no CAPSad, a maioria $64,9 \%$ (48) referiu estar em tratamento a menos de 4 meses.

Quando questionados sobre como foi o primeiro acolhimento recebido, 67,6\% (50) demanda espontânea, $29,7 \%$ (22) agendamento e 2,7\% (02) não responderam. Quanto ao acolhimento inicial ter sido individual ou coletivo, $66,2 \%$ (49) referiram individual, $32,4 \%$ (24) coletivo e $1,4 \%$ (01) não respondeu. Dos que receberam acolhimento individual, 8,1\% (06) relataram presença de acompanhante. Em relação à duração média do acolhimento recebido, 41,9\% (31) referiram duração de uma sessão, 37,8\% (28) duas sessões ou mais e $20,3 \%$ (15) não responderam.

Quanto à utilização de protocolos durante o momento do acolhimento, 51,3\% (38) afirmaram e 48,7\% (36) negaram. Dos que afirmaram, referiram que as perguntas foram de caráter investigativo e caracterização do uso de substâncias psicoativas, como na fala de U8.

"Com quantos anos comecei a beber qual tipo de bebida eu tomava quantas garrafas por dia, ou latinhas de cervejas etc." (U8).

\section{Entendimento e especificidades do acolhimento}

Ao serem questionados sobre o que entendem por acolhimento, houve diversidade de respostas, sendo possível verificar que $23,0 \%$ (17) apresentaram dificuldade em responder. Do total, 13,5\% (10) caracterizaram o momento do acolhimento como triagem e/ou consulta médica e psicológica como evidenciado na fala de U6, 10,8\% (08) momento de esclarecimento sobre o serviço e orientações sobre o tratamento exemplificado por U5, 29,7\% (22) momento de sensibilização afetiva dos profissionais como fala de U42, bem como momento de preocupação dos profissionais para com o usuário e oferta de apoio, 14,9\% (11) boa recepção no serviço e 6,7\% (5) descreveram acolhimento como espaço de relato, onde referiram receber atenção e carinho, podendo ser espaço e momento de expressão de pensamentos e emoções como verificado na fala de U67.

"Uma avaliação do profissional de saúde para ver o grau de dependência e encaminhar para o tratamento" (U6).

“Receber e orientar a respeito do tratamento" (U5).

"Acolhimento foi para mim encontro com pessoas que realmente queria me ajudar, me tirar do fundo do poço” (U42).

"Chegar no lugar, a pessoa te tratar bem, te explicar como funciona, o que oferece o tratamento e se você se encaixa" (U67).

Quando questionados sobre a influência do acolhimento para adesão ou continuidade do tratamento, 98,6\% (73) responderam positivamente. Das influências positivas destacaram: espaço de escuta das angústias e ansiedade, momento de despertar, orientação e auxílio para início e permanência no tratamento, momento de análise do comportamento decorrente do uso de drogas.

\section{Avaliação do acolhimento recebido}

Do total, 93,2\% (69) referiram satisfação pelo acolhimento recebido e não mudariam nenhum aspecto. Já $5,4 \%$ (04) referiram insatisfação e dentre as sugestões para melhora referiram: privacidade, acolhimento individual, demanda espontânea e aumento do número de profissionais disponíveis e capacitados. Ressalta-se que um usuário $(1,4 \%)$ não respondeu à questão.

"Sim, o tempo de espera que demora muito, deveria ter algo para se fazer, ser a qualquer hora não precisaria agendar" (U67).

Ainda sobre avaliação do acolhimento recebido, na resposta de U49 constata-se a importância da escuta qualificada e atenciosa, identificando e respeitando as demandas do usuário. 
"O acolhimento com cuidados especiais para pessoas que são desprezados e criticados pela sociedade, sem preconceitos. Jamais, pois no primeiro acolhimento não houve um acolhimento essencial onde eu fora tratado com respeito, receptividade. Em outros acolhimentos, não houve, cuidados, auxilio na ajuda em que eu procurava. A falta de respeito que em outros acolhimentos me trouxe fez com que eu pudesse diferenciar a importância do acolhimento" (U49).

Porém quando questionados sobre o que consideravam um acolhimento ideal, dos 93,2\% (69) que referiram satisfação, somente $81,1 \%$ (60) consideraram o acolhimento como modelo ideal. O restante $18,9 \%$ (14) referiram que o acolhimento ideal deve ter como características obrigatórias: boa receptividade do usuário pelos profissionais, ser de demanda espontânea, ser espaço seguro para expor suas reais demandas e abordar a integralidade do indivíduo.

"O ideal seria um acolhimento em todos os aspectos, devido as características, e os motivos do usuário" (U15).

"Um método de identificar as necessidades imediatas do paciente e como conviver com a dependência sem recair" (U4).

\section{DISCUSSÃO}

Os resultados apontaram a prevalência de usuários do sexo masculino que realizam tratamento nos CAPSad nas quatro cidades investigadas, corroborando com resultados de outras pesquisas similares ${ }^{12,13}$. Este dado também é confirmado pelo World Drug Report da United Nations Office on Drugs and Crime ${ }^{14}$ que mediante este fato enfatiza a necessidade do cuidado especializado com práticas terapêuticas que atendam às reais e específicas demandas de cada sexo.

Em relação ao estado civil verificou-se a predominância de usuários sem companheiros (solteiro, divorciados, viúvos) corroborando a literatura ${ }^{13,15}$. Pesquisas referem que a condição civil do usuário pode estar associada a inversão de valores de vida, pois com a instalação da dependência, o usuário passa a priorizar a busca e o uso da substância ${ }^{15}$ apresentando dificuldades em estabelecer vínculos afetivos e, principalmente, de manter seus relacionamentos frente ao surgimento e/ ou aumento de conflitos em decorrência do uso abusivo ${ }^{12}$.

Os resultados apontaram para prevalência de participantes com baixa escolaridade, aproximadamente metade da amostra, 48,6\% (36) frequentaram a escola até o ensino fundamental. Resultados também foram encontrados em outras pesquisa ${ }^{12,13,15}$. A baixa escolaridade, a cor da pele (negra ou parda), as baixas condições socioeconômicas e o desemprego ou condições desfavoráveis de trabalho são apontadas como marcadores de marginalização e exclusão social que precede o uso da substância ${ }^{16}$. Possuir uma boa escolaridade e boas condições socioeconômicas não são fatores impeditivos ao uso de substância, mas a baixa escolaridade, vulnerabilidade social e qualidade de vida precária pode intensificar as consequências do uso ${ }^{17}$.

Alguns pesquisadores referem que a baixa escolaridade também está associada ao desenvolvimento do transtorno ${ }^{15}$, pois o início precoce do uso, geralmente na adolescência, ocasiona prejuízos biopsicossociais, dentre eles, o comprometimento de sua formação educacional e profissional.

Com o desenvolvimento do processo da dependência, o usuário passa a se desinteressar pela escola e trabalho ${ }^{13,15}$ ocasionando em danos na vida acadêmica, como abandono escolar, repetições e até expulsão ${ }^{18}$. O baixo grau de escolaridade pode acarretar na carência de qualificação profissional e, portanto, dificuldades no processo de (re) inserção no mercado de trabalho formal e na valorização financeira do seu trabalho.

Frente à carência escolar, a baixa qualificação profissional e as dificuldades financeiras, muitas vezes, o usuário submete-se à prestação de serviços braçais, pouco valorizado sócio e financeiramente. Os reflexos da dependência associada à baixa escolaridade e baixa qualificação profissional são fatores que dificultam a conquista e manutenção de um trabalho e, consequente condição financeira desfavorável ${ }^{15}$. Este fato pode ser comprovado ao verificar que a maioria $75,7 \%$ (56) da amostra apresentava baixa condição financeira, repercutindo na qualidade e expectativa de vida do usuário.

Verificado prevalência de usuários que possuem religião, fato corroborado com resultados de outra pesquisa ${ }^{19}$. Pesquisas apontam que independente da religião, esta desperta e auxilia o usuário na promoção da fé, estimula a reavaliação de comportamentos, hábitos e mudanças na rotina, prevenindo o consumo de substâncias psicoativas ${ }^{19,20}$. A religiosidade possibilita melhora de sentimentos positivos, da autoestima e diminuição de sentimentos negativos, além do que o usuário passa dedicar seu tempo à realização de atividades e compromissos voltados à prática religiosa, aumentando as atividades sociais, reestruturando um novo círculo social, além de substituir as atividades relacionadas ao uso e preencher o tempo ocioso ${ }^{19,20}$.

Em relação à decisão de iniciar tratamento, a maioria dos usuários refere que foi por decisão própria. Este fato 
pode indicar a possibilidade de estes sujeitos encontraremse em período de contemplação ou ação, o que favorece o reconhecimento, mesmo que parcialmente, dos danos causados pelo uso da substância e, assim, maior probabilidade de colaborar e responsabilizar-se pelo tratamento ${ }^{16}$. Aspecto este, favorável ao tratamento no CAPSad, uma vez que a proposta de atenção deste serviço implica na participação direta, ou seja, responsabilização dos usuários em seu tratamento, à partir da participação na discussão do seu plano terapêutico em parceria com todos os atores envolvidos neste processo, como: profissionais, família, entre outros.

Ainda sobre a decisão de realizar o tratamento, evidenciou-se que parte dos usuários referiu que esta decisão foi de outras pessoas, como família e outros (justiça) e, neste caso dificilmente haverá uma mobilização inicial e/ ou envolvimento ativo do usuário no processo terapêutico.

A análise das respostas da questão referente ao entendimento dos usuários sobre o momento do acolhimento, permitiu a identificação de diferenças e diversidades de percepções dos usuários, o que pode dificultar o (re) conhecimento do acolhimento enquanto dispositivo de direito à atenção e, consequentemente falta de uso deste pelos usuários ${ }^{6}$.

A falta de informação do acolhimento pode estar associada à falha do próprio serviço na divulgação deste. $\mathrm{O}$ conceito de acolhimento é abstrato e pouco entendido como um recurso prático a ser acessado, prejudicando assim, o acesso e o aproveitamento deste enquanto direito e, também como uma das possibilidades e/ou estratégias de mobilização e sensibilização para a adesão ao tratamento.

A dificuldade de compreensão sobre o acolhimento pode acabar por esvaziá-lo de sentido, negligenciando sua especificidade, de forma a torná-lo somente uma nova denominação para procedimentos habituais como triagem ou coleta de dados iniciais ${ }^{9}$. Outro fator que pode confundir o usuário na compreensão do acolhimento é o uso de roteiros durante e, até mesmo como forma de acolhimento. Possivelmente, o uso de protocolos pode caracterizar este momento como de investigação e sobre o uso, restringindo assim, a função e objetivos do acolhimento. A utilização de roteiro pré-estabelecido pode não contemplar a singularidade e subjetividade do sujeito, características do contexto social e específico do usuário, o que dificulta a abordagem integral de atenção ao usuário. A literatura aponta que a utilização de um roteiro pré-estabelecido pode descaracterizar e simplificar a escuta qualificada, aproximando o acolhimento de uma triagem ${ }^{9}$. Acredita-se que o acolhimento deve ocorrer não só no momento de entrada do usuário ao serviço, mas durante todo o tratamento. Deve configurar-se como uma espécie de conversa, onde o profissional oferece escuta ativa às principais demandas do usuário, sendo que as informações adquiridas durante este momento podem subsidiar o preenchimento de ficha de triagem.

A percepção do acolhimento como sinônimo de triagem e/ou consulta médica e psicológica também foi apontado pelos participantes e, para discutir esta temática, é importante entender a concepção de triagem e relacionar com a proposição da atual e vigente $\mathrm{PNH}^{6}$. O termo triagem tem como significado seleção, separação de um grupo, ou escolha opondo-se aos objetivos do acolhimento que é incluir, atender às necessidades, realizar a escuta ativa das queixas, angustias e dificuldades do usuário, bem como compartilhar saberes, auxiliar na promoção da formação de vínculo e na resolutividade de problemas através da parceria usuárioprofissional, sendo, portanto, o acolhimento, uma postura ética do profissional ${ }^{6}$.

$\mathrm{O}$ acolhimento não consiste em uma triagem qualificada, mas sim na possibilidade de escuta e identificação das principais queixas e angústias do usuário, permitindo ao profissional juntamente com a equipe multiprofissional, a elaboração de intervenções voltadas ao enfrentamento destas dificuldades ${ }^{22}$. O autor ainda ressalta a importância do compartilhamento e trocas de conhecimentos entre os membros da equipe multiprofissional, evitando a centralização do saber e poder de um só profissional. O Ministério da Saúde ${ }^{6}$ ao descrever, acolhimento como momento de receptividade e ação de respeito e atenção refere que este não se resume a uma ação pontual e isolada dos processos de produção de saúde, mas sim de um conjunto de outras ações, que muitas vezes emergirão da relação humana, empática e do vínculo estabelecido entre terapeuta - paciente.

Neste sentido, a $\mathrm{PNH}^{6}$ refere que quanto ao momento e duração do acolhimento, este não deve estar somente associado ao momento de entrada do usuário no serviço, mas sim estar presente em todo processo de tratamento, devendo ser pensado como eixo central e norteador das práticas de cuidado. Desta forma, podemos pensar no acolhimento como o oposto de triagem, constituindo-se como ato de inclusão que não deve ocorrer apenas no momento da recepção, mas sim em todos os locais e etapas do serviço de saúde.

Dentro deste mesmo contexto de acolhimento como sinônimo de triagem, observou-se em algumas respostas, $\mathrm{o}$ acolhimento referenciado como a receptividade no serviço. No entanto, o acolhimento não deve se restringir apenas a uma consulta ou ao ato físico de receber o usuário no espaço do serviço, mas deva constituir-se como a receptividade ao tratamento, se compondo de uma sequência de atos e compromissos que favoreçam o vínculo do usuário em relação aos profissionais e serviço ${ }^{23}$. Ressalta-se que o acolhimento deve ser praticado em outros momentos do 
processo, como durante o atendimento individual e coletivo, momentos de recaída, alta, retorno e encaminhamento a outros dispositivos assistenciais ${ }^{23}$.

Quanto ao acolhimento coletivo, acredita-se que a oferta deste durante o processo de tratamento poderá auxiliar na identificação de usuários com risco de recaída e em sofrimento, além de contribuir para a análise e produção coletiva de estratégias de fortalecimento dos fatores de proteção, favorecendo o enfrentamento de problemas e a capacidade de auto avaliação dos riscos pelo usuário participante do grupo ${ }^{6}$. $\mathrm{O}$ acolhimento coletivo pode ser uma ferramenta eficaz na abordagem do usuário em outros espaços da rede de atendimento além do CAPSad, como em unidades básicas de saúde 9 . Também é importante enfatizar a prática do acolhimento coletivo como processo grupal de educação preventiva ${ }^{24}$.Porém, $o$ acolhimento coletivo tem como características negativas, a possibilidade dos usuários sentirem-se constrangidos, expostos e tendo sua privacidade e intimidade violadas, além de não disponibilizar de um momento para adequada avaliação dos riscos inevidentes e da vulnerabilidade de cada indivíduo.

A concepção de acolhimento dos usuários que o definiram como esclarecimento sobre o serviço e orientações sobre o tratamento contempla em partes o que seria o acolhimento ideal pela $\mathrm{PNH}^{6}$, visto que ele também tem a função de esclarecimento de dúvidas e orientações em relação à melhor forma de lidar com o tratamento. Porém, há a necessidade de destacar que o acolhimento deve ir além, devendo constituir-se em relações humanas presentes durante todo o tratamento, dando espaço para que o paciente seja ouvido e orientado conforme suas demandas e angústias, considerando a singularidade e integralidade deste.

A percepção do acolhimento como momento de respeito e atenção do profissional para com o usuário aponta para a existência de relações empáticas e de aproximação dos profissionais na oferta de ajuda e apoio, ou como oferta de momento para expressão de sentimentos e emoções. Neste sentido, o papel do profissional é para além de oferecer escuta qualificada, identificação de riscos e vulnerabilidade, mas de empatia e solidariedade com o usuário, compreendendo sua queixa e em parceria com o usuário encontrar estratégias para resolutividade do problema ${ }^{22}$.

Os usuários ao buscar tratamento geralmente apresentam sentimentos ambivalentes e sensibilidade quanto à maneira como são recebidos ${ }^{21}$. Neste sentindo, a sensibilização afetiva, a escuta empática e boa recepção são elementos fundamentais no acolhimento, pois auxilia no processo de adesão ao tratamento e da formação de vínculo do usuário com o profissional e serviço ${ }^{6,9}$.

Os resultados apontaram para aceitação e satisfação do acolhimento que lhe é oferecido, porém mediante controvérsias de respostas de outras questões, faz-se importante discutir sobre possiblidade de conformismo mediante o predomínio de sentimentos de menos valia e culpa, vulnerabilidade social, fragilidade de aspectos cognitivos e de valores sociais e, principalmente a falta de (re)conhecimento dos usuários em relação aos seus direitos, motivos estes que podem levar o usuário a considerar que $\mathrm{o}$ acolhimento ofertado já pode ser considerado ideal.

Número expressivo de usuários reconhece e afirma a influência positiva do acolhimento para a adesão, sucesso e continuidade do seu tratamento. $\mathrm{O}$ acolhimento quando norteado por atitudes de respeito, horizontalidade e empatia, promovem o sentimento de confiança, troca de informações e saberes e, consequente início da formação do vínculo terapeuta-paciente, possibilitando assim o cuidado integral e humanizado ao usuário ${ }^{23}$. Acredita-se que o acolhimento e a construção de vínculo permitem que o usuário exponha suas reais demandas, aspecto este decisivo na relação de cuidado entre o profissional e o usuário, incidindo na resolutividade dos problemas apresentados, além de possibilitar a corresponsabilização de ambos.

Dentre as mudanças apontadas para a melhora e aprimoramento do acolhimento, destacou-se a disponibilidade e capacitação dos profissionais, sessões individuais e ser demanda espontânea. Nota-se que essas problemáticas se dão pela demanda de acolhimento versus a quantidade de profissionais disponíveis, o que leva a reflexão sobre a necessidade de contratação de profissionais, evitando a sobrecarga dos profissionais envolvidos, o que prejudica na realização da prática integral do acolhimento.

Atualmente a maioria dos serviços da rede de saúde funciona com o quadro mínimo de profissionais exigidos, culminando quase sempre, no estresse e sobrecarga de trabalho e, destacam ainda que, o profissional também tem que estar preparado emocionalmente para ouvir as queixas e angústias relacionadas ao sofrimento do usuário9. Destaca-se também a importância da capacitação e o treinamento destes profissionais, que na realidade cotidiana enfrentam enormes dificuldades na tentativa de articular a teoria com a prática ${ }^{25}$. A presença de um serviço de portas abertas com práticas de atenção integral e cuidado humanizado ao usuário, sempre respaldadas no acolhimento dos profissionais, tornam-se vitais e imprescindíveis para a possibilidade de criação de expectativas e esperança de vida dos usuários de substâncias.

\section{CONSIDERAÇÕES FINAIS}

Os resultados obtidos apontam as diversidades e diferentes percepções dos usuários acerca do acolhimento recebido, podendo restringir o uso deste em sua totalidade 
Silva M, et al. A prática do acolhimento na atenção ao usuário de substâncias psicoativas. Rev Ter Ocup Univ São Paulo. 2019 jan./abr.;30(1):1-9.

de direito. Para que isto não ocorra, faz-se necessário o desenvolvimento pelos profissionais, de ações e politicas informativas ao usuário sobre o dispositivo, além da formação e capacitação de profissionais habilitados e sensíveis, familiarizados com os princípios de humanização e de atenção psicossocial. A pesquisa possibilitou aprofundamento sobre $\mathrm{o}$ modo como tem se dado o acolhimento do usuário e a influência positiva deste para a adesão, sucesso e continuidade do tratamento, principalmente por se constituir como possibilidade de criação de vínculo entre profissionais e serviço.

Contribuição dos autores: Silva ML e Marucci LD participaram de integralmente de todas as etapas do trabalho e Guimarães CS participou das etapas de concepção e análise dos dados. Ressalta-se que todos os autores aprovaram a versão final do artigo.

Conflito de Interesse: Inexistência de conflito de interesse.

Fonte de Financiamento: Este trabalho teve financiamento parcial do Conselho Nacional de Desenvolvimento Científico e Tecnológico (CNPq) por meio de bolsas de iniciação científica de LD Marucci e CS Guimarães.

\section{REFERÊNCIAS}

1. Brasil. Ministério da Saúde. Portaria n ${ }^{\circ} 3.088$, de 23 de dezembro de 2011. Institui a Rede de Atenção Psicossocial para pessoas com sofrimento ou transtorno mental e com necessidades decorrentes do uso de crack, álcool e outras drogas, no âmbito do Sistema Único de saúde (SUS). Diário Oficial da União, Poder Executivo, Brasília, DF, 21 maio 2013.

2. Brasil. Ministério da Saúde. Ministério da Saúde. Secretaria de Atenção à Saúde. A política do Ministério da Saúde para atenção integral a usuários de álcool e outras drogas. 2a. ed. Brasília; 2004.

3. Brasil. Ministério da Saúde. Portaria ${ }^{\circ} 1.028$ de 01 de julho de 2005. Determina que as ações que visam à redução de danos sociais e à saúde, decorrentes do uso de produtos, substâncias ou drogas que causem dependência, sejam reguladas por esta Portaria. Diário Oficial da União, Poder Executivo, Brasília, DF, 1 jul. 2005.

4. Brasil. Conselho Nacional de Políticas Sobre Drogas. Resolução CONAD n ${ }^{\circ}$ 01, de 09 de março de 2018. Define as diretrizes para o realinhamento e fortalecimento da PNAD, Plano Nacional Sobre Drogas, aprovada pelo Decreto 4345, de 26 de agosto de 2002. Brasília, DF; 2018.

5. Brasil. Ministério da Saúde. Secretaria de Atenção à Saúde. Departamento de Ações Programáticas Estratégicas. Guia estratégico para o cuidado de pessoas com necessidades relacionadas ao consumo de álcool e outras drogas: Guia AD. Brasília, DF; 2015.

6. Brasil. Ministério da Saúde. Secretaria de Atenção à Saúde. Núcleo Técnico da Política Nacional de Humanização. Acolhimento nas práticas de produção de saúde. 2a. ed. Brasília, DF; 2006.

7. Ferreira ACZ, Capistrano FC, Souza EB, Borba LO, Kalinke LP, Maftum MA. Motivações de dependentes químicos para o tratamento: percepção de familiares. Rev Bras Enferm. 2015;68(3):474-81. https://doi.org/10.1590/0034$7167.2015680314 \mathrm{i}$.

8. Greco C. O grupo de acolhimento: um dispositivo para facilitar a adesão ao tratamento. Campinas: Ed. UNICAMP; 2009.

9. Salles DB, Silva ML. Percepção de profissionais da área de saúde mental sobre o acolhimento ao usuário de substância psicoativa em CAPSad. Cad Bras Ter Ocup. 2017;25(2):341-9. https://doi.org/10.4322/0104-4931.ctoAO0803.

10. Silva CR, Santos CN, Nogueira JN, Malfitano APS. Mapeamento da atuação do terapeuta ocupacional nos Centros de Atenção Psicossocial Álcool e Drogas (CAPS ad) do interior do estado de São Paulo. Cad Ter Ocup UFSCar. 2015;23(2):321-34. https://doi.org/10.4322/0104-4931. ctoAO0504.

11. Bardin L. Análise de conteúdo. São Paulo: Edições 70; 2011.

12. Cantão L, Botti NCL. Comportamento suicida entre dependentes químicos. Rev Bras Enferm. 2016;69(2):38996. http://dx.doi.org/10.1590/0034-7167.2016690224i.

13. Monteiro CFS, Fé LCM, Moreira MAC, Albuquerque IEM, Silva MG, Passamani MC. Perfil sociodemográfico e adesão ao tratamento de dependentes de álcool em CAPS-ad do Piauí. Esc Anna Nery. 2011;15(1):90-5. http://dx.doi.org/10.1590/ S1414-81452011000100013

14. United Nations Office on Drugs and Crime (UNODC): World Drug Report. New York: Unodc; 2016. Available from: http://www.unodc.org/doc/wdr2016/WORLD_DRUG_ REPORT_2016_web.pdf.

15. Capistrano FC, Ferreira ACZ, Silva TL, Kalinke LP, Maftum MA. Clinical sociodemographic profile of chemically dependents under treatment: record analysis. Esc Anna 
Nery. 2013;17(2):234-41. http://dx.doi.org/10.1590/S141481452013000200005 .

16. Souza J. Crack e exclusão social. Brasília, DF: Ministério da Justiça e Cidadania; 2016. Disponível em: https://obid. senad.gov.br/livro-crack-e-exclusao-social_digital_web.pdf.

17. Brasil. Ministério da Justiça. Secretaria Nacional de Políticas sobre DROGAS. Uma política sobre drogas é uma política sobre pessoas. Brasília, DF; 2016. Disponível em: http://www.justica.gov.br/ news/senad-mj-disponibiliza-relatorio-uma-politica-sobre-drogase-uma-politica-sobre-pessoas/senad-relatorio-e-anexo-1.pdf.

18. Martins M, Santos MA, Pillon SC. Percepções de famílias de baixa renda sobre o uso de drogas por um de seus membros. Rev Latino-Am Enfermagem. 2008;16(2):293-8. http://dx.doi. org/10.1590/S0104-11692008000200019.

19. Bettarello VC, Silva LMA, Molina NPFM, Silveira T, Rodrigues LR. Qualidade de vida, espiritualidade, religião e crenças pessoais de dependentes químicos em tratamento. Rev Elet Enf. 2016;18. https://doi.org/10.5216/ree.v18.41677.

20. Silva ML, Guimarães CF, Salles DB. Fatores de risco e proteção à recaída na percepção de usuários de substâncias psicoativas. Rev Rene. 2014;15(6):1007-15. https://doi. org/10.15253/2175-6783.2014000600014.
21. Sousa PF, Ribeiro, LCM, Melo JRF, Maciel SC, Oliveira MX. Dependentes químicos em tratamento: um estudo sobre a motivação para mudança. Temas Psicol. 2013;21(1):259-68. https://doi.org/10.9788/TP2013.1-18.

22. Solla JJSP. Acolhimento no sistema municipal de saúde. Rev Bras Saude Mater Infant. 2005;5(4):493-503. https://doi. org/10.1590/S1519-38292005000400013.

23. Jorge MSB, Pinto DM, Quinderé PHD, Pinto AGA, Sousa FSP, Cavalcante CM. Promoção da saúde mental: tecnologias do cuidado: vínculo, acolhimento, co-responsabilização de autonomia. Ciênc Saúde Coletiva. 2009;16(7):3051-60. https://doi.org/10.1590/S1413-81232011000800005.

24. Azambuja MPR, Debastiani C, Duarte CC, Minozzo F, Souza AC. Relato de experiência: o acolhimento em grupo como uma estratégia para a integralidade. PsicoUSF, 2007;12(1):121124. Disponível em: http://www.scielo.br/pdf/pusf/v12n1/ v12n1a14.pdf.

25. Mângia EF, Castilho JPLV, Duarte VRE. A construção de projetos terapêuticos: visão de profissionais em dois centros de atenção psicossocial. Rev Ter Ocup Univ São Paulo. 2006;17(2):87-98. https://doi.org/10.11606/issn.2238-6149. v17i2p87-98. 